\title{
Use of cod liver oil during pregnancy associated with lower risk of Type I diabetes in the offspring
}

\author{
L. C. Stene ${ }^{1}$, J. Ulriksen ${ }^{2}$, P. Magnus ${ }^{1}$, G. Joner ${ }^{3}$ \\ ${ }^{1}$ Section of Epidemiology, National Institute of Public Health, Oslo, Norway \\ ${ }^{2}$ Department of Paediatrics, Vest-Agder Central Hospital, Kristiansand, Norway \\ ${ }^{3}$ Aker Diabetes Research Centre, Aker University Hospital, Oslo, Norway
}

\section{Abstract}

Aims/hypothesis. To test whether cod liver oil or vitamin D supplements either taken by the mother during pregnancy or by the child in the first year of life is associated with lower risk of Type I (insulin-dependent) diabetes mellitus in children.

Methods. We carried out a population-based case control study in Vest-Agder county of Norway, evaluating the use of supplements by a mailed questionnaire. We received responses from 85 diabetic subjects and 1071 control subjects. Odds ratios (OR) with $95 \%$ confidence intervals $(\mathrm{CI})$ were estimated using logistic regression analyses.

Results. When mothers took cod liver oil during pregnancy their offspring had a lower risk of diabetes. The unadjusted OR was $0.30,95 \%$ CI: (0.12 to 0.75 ), $p=0.01$. This association changed very little and was still significant after adjusting for age, sex, breast- feeding and maternal education. Mothers taking multivitamin supplements during pregnancy [adjusted $\mathrm{OR}=1.11,95 \% \mathrm{CI}:(0.69$ to 1.77$)]$, infants taking cod liver oil in the first year of life [adjusted $\mathrm{OR}=0.82,95 \% \mathrm{CI}$ : (0.47 to 1.42$)$ and the use of other vitamin D supplements in the first year of life [adjusted OR $=1.27,95 \% \mathrm{CI}$ : $(0.70$ to 2.31$)$ ] was significantly associated with the risk of diabetes.

Conclusion/interpretation. We found that cod liver oil taken during pregnancy was associated with reduced risk of Type I diabetes in the offspring. This suggests that vitamin $\mathrm{D}$ or the $n-3$ fatty acids eicosapentaenoic acid and docosahexaenoic acid in the cod liver oil, or both, have a protective effect aginst Type I diabetes. [Diabetologia (2000) 43: 1093-1098]

Keywords Type I (insulin-dependent) diabetes mellitus, diet, vitamin D, case control study, aetiology.
Type I (insulin-dependent) diabetes mellitus results from the destruction of the insulin-producing beta cells of the pancreas, a disease process that can last several years before the clinical onset of the disease [1]. Type I diabetes is a multifactorial disease but the factors initiating and propagating the process leading to clinical disease are mostly not known. Genetic factors in the HLA complex play an important part in its

Received: 19 April 2000 and in revised form: 6 June 2000

Corresponding author: L.C. Stene, Section of Epidemiology, National Institute of Public Health, P. O. Box 4404 Torshov, $\mathrm{N}-0403$ Oslo, Norway

Abbreviations: OR, Odds ratio; EPA, eicosapentaenoic acid; DHA, docosahexaenoic acid. pathogenesis, probably together with environmental factors such as diet or virus infections $[1,2]$. The hypothesis proposing that breastfeeding could reduce the risk of Type I diabetes [3] has stimulated studies focusing on the possible causal role of infant diet in Type I diabetes. The role of breastfeeding and early introduction of cows' milk is still controversial [4-6] but there are good reasons to believe that environmental risk factors could operate early in life, possibly in utero [7]. Recently, knowledge of untraditional effects of vitamin D has been applied to Type I diabetes [8]. Vitamin D has several effects on the immune system which could be of potential relevance in the pathogenesis of Type I diabetes [8-11] and has been shown to protect against autoimmune diabetes in experimental animals $[12,13]$. Furthermore, a previous 
multicentre case control study has shown that vitamin $\mathrm{D}$ supplements taken in the first year of life is associated with reduced risk of Type I diabetes [14].

Vitamin D is produced in the skin when exposed to ultra-violet radiation from sunlight [15]. Exposure to sunlight in northern countries is, however, below optimal for infants and possibly for pregnant women [16]. In Norway, dietary vitamin D supplement is recommended from infancy, preferably in the form of cod liver oil [17]. This is mainly to prevent vitamin D deficiency that causes rickets. Cod liver oil is specifically recommended because it also contains the long-chain $n-3$ fatty acids eicosapentaenoic acid (EPA) and docosahexaenoic acid (DHA) that have been regarded to be essential for infants $[17,18]$. Dietary long-chain $n-3$ fatty acids are incorporated in cell membranes and have anti-inflammatory effects which could be potentially relevant in the aetiology of Type I diabetes $[19,20]$.

Because breast-milk is a relatively poor source of vitamin $\mathrm{D}$, the vitamin $\mathrm{D}$ status of the newborn child is dependent on stored vitamin $\mathrm{D}$ acquired from the mother in utero or dietary supplements before weaning [15]. The vitamin D intake of the mother during pregnancy is reflected by the vitamin D status of the newborn child $[16,21,22]$. Therefore, if vitamin $\mathrm{D}$ has a protective effect against Type I diabetes in children, it is possible that the mothers' use of vitamin D supplements during pregnancy is associated with a reduced risk of Type I diabetes in their offspring. The objective of our study was to estimate the associations between the use of cod liver oil or other vitamin D supplements, either by the mother during pregnancy or by the child in the first year of life and the risk of childhood-onset Type I diabetes in the children.

\section{Subjects and methods}

Subjects. In Norway, all patients newly diagnosed with Type I diabetes before the age of 15 have been registered in the National Childhood Diabetes Registry with a high level of ascertainment between 1973 and 1982 [23]. Since 1 January 1989, all new cases of diabetes have been prospectively registered, still with a high level of ascertainment [24]. We followed the diagnostic criteria of EURODIAB [25]. Based on this prospective registration, we designed a population-based case control pilot study in Vest-Agder county of Norway. Vest-Agder is the southernmost county of Norway, with a population of 154000 in 1998 . This corresponds to $3.5 \%$ of the population of Norway. All patients diagnosed with Type I diabetes before the age of 15 in Vest-Agder county between 1989 and 1998 and who were born between 1982 and 1998 were eligible to participate. Control subjects were selected at random from the official population register among residents in Vest-Agder county at the end of 1998 who were born between 1982 and 1998. All participants were contacted by mail in May 1999. After one reminder, 85 diabetic subjects $(94.4 \%$ of those invited) and 1071 control subjects ( $72.7 \%$ of those invited) had responded. Written informed consent was obtained from the parents of all participants and from all participants able to write. The study protocol was approved by the Regional Ethics Committee and by the National Data Inspectorate.

Evaluation of dietary supplements and potential confounders. Diabetic and control subjects received a four-page questionnaire by mail. The questionnaire included general questions on sex, age, and on the children's health during childhood and the mother's health during pregnancy. The participants were informed that the causes of childhood diabetes are mostly not known and that we were studying possible causes of diabetes. The mother of the index child was asked to complete the questionnaire, preferably together with the father and the index child. The questionnaire asked whether the participant's mother took cod liver oil or vitamin/mineral supplements during pregnancy with the participant. A negative or affirmative answer could be ticked for cod liver oil, multivitamin and other (to be specified). In a section devoted to the participant's dietary habits in the first year of life, the questionnaire asked whether the participant received cod liver oil or other vitamin D supplements during the first year of life. A negative or affirmative answer could be ticked for cod liver oil, multivitamin (with examples of the most common brand names) and other (to be specified). Those who ticked the affirmative for cod liver oil or vitamin supplement were considered exposed to the respective factor. Those who specified another preparation containing vitamin D were also considered exposed to vitamin $\mathrm{D}$ supplement. The questionnaire also contained questions on the duration of exclusive breastfeeding and maternal educational level at the time of birth of the index child.

Data analysis. The data from all questionnaires were optically scanned and checked for errors. We used SPSS for Windows, release 9.0 for data analysis. Information was missing for one diabetic $(1.2 \%)$ and 24 control subjects $(2.2 \%)$ on the mother's use of cod liver oil or multivitamin supplements during pregnancy and for 7 diabetic $(8.2 \%)$ and 91 control subjects $(8.5 \%)$ on the use of cod liver oil or other vitamin D supplements in the first year of life. These were excluded from the respective analyses. Odds ratios (OR) with $95 \%$ confidence intervals (CI) associated with each exposure were estimated using logistic regression, adjusting for age and other potential confounding factors [26]. An OR less than 1.0 indicates a negative association, an OR more than 1.0 a positive association and an OR of 1.0 a lack of association. The relation between age (at the time of data collection) and risk of Type I diabetes was approximately logit-linear and age was entered into the logistic model as a continuous variable. Separate analyses stratified by age group in four categories were also done. Breastfeeding practices and maternal education were adjusted for by entering them into the logistic model as binary variables (exclusive breastfeeding $<3$ months vs $\geq 3$ months and maternal education $\geq 12$ years vs $<12$ years). For evaluation of one supplement (e.g. cod liver oil), use of other supplements (e.g. multivitamin) was also evaluated as a confounder. Analyses were also adjusted for sex. A two-sided significance level less than 0.05 was regarded as statistically significant. With the given sample size, we had $52 \%$ power to obtain a significant result for exposures with a prevalence of $20 \%$ among control subjects if the true OR was 0.5 , and $84 \%$ power if the true OR was 0.3 . 
Table 1. Distribution of age, sex, breastfeeding practice and maternal education for subjects with Type I diabetes and population controls selected at random in Vest-Agder county, Norway (SD standard deviation)

\begin{tabular}{|c|c|c|c|}
\hline Variable & Cases $(n=85)$ & Controls $(n=1071)$ & Odds ratio $(95 \% \mathrm{CI})$ \\
\hline $\operatorname{Age}^{\mathrm{a}}($ mean, SD) & $11.1(3.6)$ & $8.5(4.7)$ & \\
\hline Male sex & $54(63.5 \%)$ & $522(48.7 \%)$ & $1.8(1.2,2.9)$ \\
\hline Exclusive breastfeeding $<3$ months $^{\mathrm{b}}$ & $22(25.9 \%)$ & $258(24.2 \%)$ & $1.1(0.7,1.8)$ \\
\hline
\end{tabular}

a Age at data collection

$\mathrm{b}$ There were four controls with missing information for breastfeeding practice
${ }^{\mathrm{c}}$ Highest attained education at birth of the index child (there were one case and 17 controls with missing information for maternal education)

Table 2. Estimated odds ratios for Type I diabetes in children associated with mothers' use of dietary supplements during pregnancy

\begin{tabular}{|c|c|c|c|c|}
\hline \multirow{2}{*}{$\begin{array}{l}\text { Type of supplement used } \\
\text { by mother in pregnancy }\end{array}$} & \multirow{2}{*}{$\begin{array}{l}\text { Cases } \\
(n=84)\end{array}$} & \multirow{2}{*}{$\begin{array}{l}\text { Controls } \\
(n=1047)\end{array}$} & \multicolumn{2}{|c|}{ Odds ratio $(95 \% \mathrm{CI})$} \\
\hline & & & Unadjusted & Adjusted $^{c}$ \\
\hline \multicolumn{5}{|l|}{ Cod liver oil } \\
\hline No & 79 & 865 & & \\
\hline \multicolumn{5}{|l|}{ Multivitamin } \\
\hline Yes & 32 & 388 & $1.05(0.66,1.65)$ & $1.11(0.69,1.77)$ \\
\hline No & 52 & 659 & & \\
\hline
\end{tabular}

${ }^{\mathrm{a}} p=0.01 ;{ }^{\mathrm{b}} p=0.03$

c Adjusted for age, sex, breastfeeding, maternal education and 'other supplement' (use of cod liver oil adjusted for use of

\section{Results}

Background variables. On average the diabetic were older than the control subjects (mean age 11.1 vs 8.5 years). There were more males among diabetic than among control subjects (Table 1). The proportion of participants who had mothers with a high level of education was slightly but not statistically significantly lower among diabetic than among control subjects.

Maternal use of supplements in pregnancy. We found a strong negative association between mothers taking cod liver oil during pregnancy and the risk of Type I diabetes in their children, with an OR of 0.3 (Table 2). This association was only slightly changed and still significant after adjustment for age, sex, maternal use of multivitamins, duration of exclusive breastfeeding and mothers' educational level. There was no significant association between the mothers' use of multivitamins during pregnancy and the risk of Type I diabetes, neither crude nor after adjustment for possible confounders. Odds ratios were homogeneous in all age groups (data not shown). Because a few mothers had used both cod liver oil and multivitamins during pregnancy, an analysis considering the single and combined effects of cod liver oil and multivitamins was done. The odds ratio for each type of supplement alone and that for using both types of supplements together were estimated relative to using neither (Table 3 ). This showed that the multivitamin and vice versa). Six individuals were excluded due to missing information on maternal education or breastfeeding

association between taking of cod liver oil during pregnancy and the risk of diabetes was independent of taking multivitamin supplements duringpregnancy.

Use of supplements in the first year of life. In the first year of life neither use of cod liver oil nor use of other vitamin D supplements showed statistically significant associations with the risk of diabetes (Table 4). Because a few children had used both cod liver oil and other vitamin D supplements in the first year of life, an analysis was done to estimate the single and combined associations (Table 5). This indicated a possible negative association with cod liver oil alone (adjusted OR $=0.45$ ) and had a complete lack of association with other vitamin D supplements (adjusted $\mathrm{OR}=1.00$ ). There was some variation in the size of the odds ratios in different age groups, but this was not systematic and not statistically significant (data not shown).

\section{Discussion}

In Norway cod liver oil is an important dietary source of vitamin D [27] and the long-chain $n$-3 fatty acids eicosapentaenoic acid (EPA) and docosahexaenoic acid (DHA) [28]. The status of vitamin D [15,16,22] and of EPA and DHA [18] in the mother during pregnancy is reflected by the status of these nutrients in the newborn child. 
Table 3. Analysis of single and joint effects of mothers' taking cod liver oil and multivitamin supplements during pregnancy on the risk of childhood-onset Type I diabetes in the children

\begin{tabular}{|c|c|c|c|c|}
\hline \multirow{2}{*}{$\begin{array}{l}\text { Type of dietary supplement used } \\
\text { by mother in pregnancy }\end{array}$} & \multirow{2}{*}{$\begin{array}{l}\text { Cases } \\
(n=84)\end{array}$} & \multirow{2}{*}{$\begin{array}{l}\text { Controls } \\
(n=1047)\end{array}$} & \multicolumn{2}{|c|}{ Odds ratio $(95 \% \mathrm{CI})$} \\
\hline & & & Unadjusted & Adjusted $^{\mathrm{a}}$ \\
\hline None & 49 & 551 & 1.00 ( reference ) & $1.00($ reference $)$ \\
\hline Multivitamin & 30 & 314 & $1.07(0.67,1.73)$ & $1.12(0.69,1.83)$ \\
\hline Cod liver oil and multivitamin & 2 & 74 & $0.30(0.07,1.28)$ & $0.36(0.08,1.53)$ \\
\hline
\end{tabular}

a Adjusted for age, sex, breastfeeding and maternal education. Six individuals were excluded from the adjusted analysis due to missing information on maternal education or breastfeeding

Table 4. Estimated odds ratios for Type I diabetes associated with taking cod liver oil and other vitamin D supplements in the first year of life

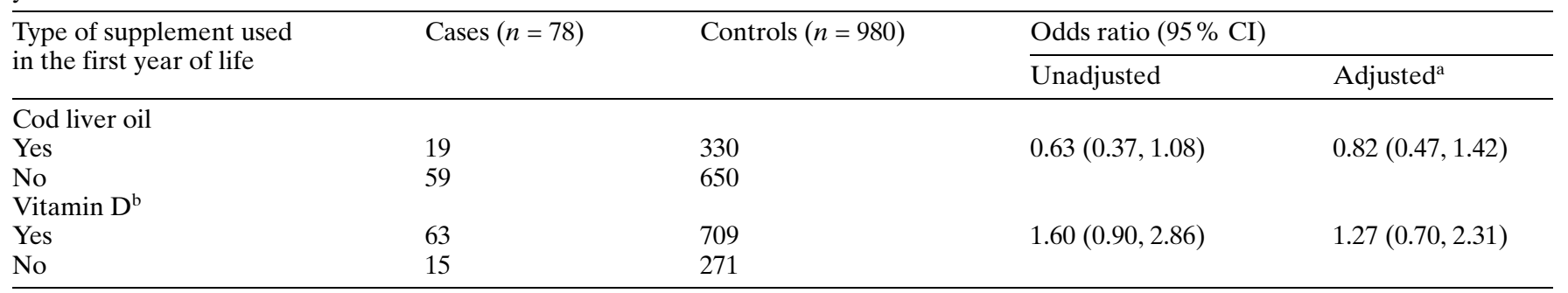

a Adjusted for age, sex, breastfeeding, maternal education and 'other supplement' (use of cod liver oil adjusted for use of mul-

${ }^{\mathrm{b}}$ May contain other vitamins in addition to vitamin $\mathrm{D}$ tivitamin and vice versa)

We did not observe a statistically significant association between the mothers' use of multivitamin supplements during pregnancy and the risk of diabetes in their children. Assuming that vitamin $\mathrm{D}$ was responsible for the association between the mothers' taking cod liver oil and the risk of diabetes in children, this could seem inconsistent. A possible explanation is that the bioavailability of vitamin D could be better from cod liver oil than from other supplements. In a prospective study of more than 300 women and their children born in Oslo, it was found that newborn children of mothers who had taken cod liver oil during pregnancy had statistically significantly higher concentrations of 25-(OH)-vitamin D in the cord blood than children of mothers who had taken other vitamin D supplements during pregnancy (Gry Hay et al., Institute for Nutrition Research, University of Oslo, unpublished data). It should be added, however, that the pregnant women taking cod liver oil in that study took amounts providing statistically significantly higher doses of vitamin $\mathrm{D}$ than that provided from other vitamin D supplements in those who took other supplements during pregnancy. Another possible explanation is that there was some misclassification of intake of vitamin D based on the multivitamin information. Although most multivitamin supplements commercially available in Norway contain vitamin $\mathrm{D}$, not all the participants in our study specified the type of multivitamin supplement they had used. According to nutritionists experienced in interview-based assessments of dietary intake, the concept of multivitamin supplements is not clear to some people, whereas the use of cod liver oil is always easy to estimate. In addition, because our data are compatible with an effect of cod liver oil and not with other vitamin D supplements, we must consider the possibility that constituents in cod liver oil other than vitamin $\mathrm{D}$ are responsible for the negative association with Type I diabetes in children, perhaps in combination with vitamin D. Both EPA and DHA have anti-inflammatory properties of potential relevance in the pathogenesis of Type I diabetes. For instance, supplementation with dietary long-chain $n-3$ fatty acids has been shown to decrease the production of interleukin-1 and tumour necrosis factor by human mononuclear cells [19] and to decrease the expression of HLA class II molecules on activated human monocytes [20]. An effect of marine fatty acids is also compatible with the observation of a statistically significantly lower incidence of Type I diabetes in municipalities in Norway which are fisheries than in the rest of the country [29]. Although there is little direct evidence from previous studies in humans or animals to support a protective role of long-chain $n-3$ fatty acids in the pathogenesis of Type I diabetes, this possibility should be considered.

We found that the associations between taking cod liver oil or other vitamin D supplements in the first year of life and the risk of Type I diabetes were not statistically significant. Stratification by use of other supplements (Table 5) indicated a tendency for a negative association between taking cod liver oil in the 
Table 5. Analysis of single and joint effects of use of cod liver oil and other vitamin D supplements in the first year of life on the risk of childhood-onset Type I diabetes

\begin{tabular}{|c|c|c|c|c|}
\hline \multirow{2}{*}{$\begin{array}{l}\text { Type of supplement used } \\
\text { in the first year of life }\end{array}$} & \multirow[t]{2}{*}{ Cases $(n=78)$} & \multirow[t]{2}{*}{ Controls $(n=980)$} & \multicolumn{2}{|c|}{ Odds ratio $(95 \% \mathrm{CI})$} \\
\hline & & & Unadjusted & Adjusted $^{\mathrm{b}}$ \\
\hline None & 11 & 139 & 1.00 (reference) & 1.00 (reference) \\
\hline Vitamin $\mathrm{D}^{\mathrm{a}}$ & 48 & 511 & $1.19(0.60,2.35)$ & $1.00(0.50,2.00)$ \\
\hline Cod liver oil and vitamin D & 15 & 198 & $0.96(0.43,2.15)$ & $0.98(0.43,2.23)$ \\
\hline
\end{tabular}

${ }^{a}$ May contain other vitamins in addition to vitamin D

${ }^{\mathrm{b}}$ Adjusted for age, sex, breastfeeding and maternal education.

Six individuals were excluded from the adjusted analysis

due to missing information on maternal education or breastfeeding

first year of life and the risk of diabetes and a lack of such an association for other vitamin D supplements. Although speculative, this suggests a similar relation between cod liver oil and other supplements taken by the child in the first year of life to that of the mother taking those supplements during pregnancy, and the above mentioned possible explanations could also be relevant here. A large multicentre case control study in seven European countries, found that vitamin D supplements taken in the first year of life was significantly associated with reduced risk for childhood Type I diabetes $(\mathrm{OR}=0.67)$ but with some differences between the centres [14]. No conclusion could, however, be drawn from our data on a possible relation between the use of vitamin D supplements in the first year of life and the risk of Type I diabetes.

A number of precautions are warranted when making inferences from the data of this study. Although the negative association between the mothers' use of cod liver oil and diabetes in their children was strong and statistically significant, the absolute number of those exposed to the supplements was small and the confidence intervals for the odds ratios were relatively wide. The assessment of vitamin D and cod liver oil intake was relatively crude. We did not attempt to estimate the amount or duration of supplementation. Because the mean dietary intake of vitamin D among pregnant women when excluding supplements is approximately a third of that provided by the recommended daily dose of cod liver oil and other supplements [30], there is good reason to believe that those who took supplements had a higher intake of vitamin D than those who did not. A similar argument is probably valid for the intake of the $n-3$ fatty acids EPA and DHA in cod liver oil. In our study, we selected two exposure windows for the children, in utero and in the first year of life, respectively. The first year of life was also used in the EURODIAB study [14] and roughly corresponds to the period when children are most prone to vitamin $\mathrm{D}$ deficiency [15]. In addition, our selected exposure windows are in accordance with the concept that the effect of environmental factors in the development of Type I diabetes is important early in life, possibly in utero [7].
The time period of exposure to vitamin D, EPA and DHA that is biologically most relevant in our context is not known but our data suggest that exposure in utero could be relevant for the development of Type I diabetes.

There could have been some degree of misclassification of exposure due to inaccurate recall and the possibility of recall bias should be considered. If recall bias was responsible for the observed association between the mothers' use of cod liver oil and the risk of diabetes, we would expect the association to be stronger among those whose length of recall was longest (assuming homogeneous effect over age groups). The strength of association was, however, homogeneous in different age groups. Furthermore, we would expect the extent of differential recall between mothers of diabetic and those of control children to be similar for use of cod liver oil and multivitamins during pregnancy but there was no association with multivitamins. In addition, the possible association of vitamin D or cod liver oil with Type I diabetes is not known by the public. It therefore seems unlikely that recall bias is responsible for the majority of the observed association between the mothers' use of cod liver oil and the risk of diabetes in their children.

Although the analyses were adequately adjusted for age, sex, duration of breastfeeding and maternal education, we cannot rule out the possibility that other extraneous factors are associated with both the use of cod liver oil and the low risk of Type I diabetes. It is, however, difficult to envisage other factors that could have a sufficiently strong association with both exposure and disease to account for the observed relation.

Our study showed a negative association between use of cod liver oil in pregnancy and the subsequent risk of Type I diabetes in the children before they reach 15 years of age. Plausible biological mechanisms could explain a potential causal effect of vitamin $\mathrm{D}$ or long-chain $n-3$ fatty acids present in the cod liver oil, or both.

Acknowledgements. G. Joner and L.C. Stene were supported by a grant from the Norwegian Foundation for Health and Re- 
habilitation (grant no 1997/156). Funding was also kindly provided by TINE Norwegian Dairies and Novo Nordisk Pharma A/S. We are grateful for the assistance and support from research nurse U.T. Hinna, Dr P. Vesterhus, Professor O. Søvik and the Norwegian Childhood Diabetes Study Group.

\section{References}

1. Atkinson MA, Maclaren NK (1994) The pathogenesis of insulin-dependent diabetes mellitus. $\mathrm{N}$ Engl J Med 24: 1428-1436

2. Nerup J, Mandrup-Poulsen T, Helqvist S et al. (1994) On the pathogenesis of IDDM. Diabetologia 37 [Suppl 2]: S82-S89

3. Borch-Johnsen K, Joner G, Mandrup-Poulsen $\mathrm{T}$ et al. (1984) Relation between breast-feeding and incidence rates of insulin-dependent diabetes mellitus: a hypothesis. Lancet ii: 1083-1086

4. Åkerblom HK, Knip M (1998) Putative environmental factors in Type 1 diabetes. Diabetes Metab Rev 14: 31-67

5. Atkinson MA, Ellis TM (1997) Infants diets and insulindependent diabetes: evaluating the "cows' milk hypothesis" and a role for anti-bovine serum albumin immunity. J Am Coll Nutr 16: 334-340

6. Scott FW, Kolb H (1996) Cow's milk and insulin-dependent diabetes mellitus. Lancet 348: 613

7. Leslie DG, Elliott RB (1994) Early environmental events as a cause of IDDM. Diabetes 43: 843-850

8. Mauricio D, Mandrup-Poulsen T, Nerup J (1996) Vitamin $\mathrm{D}$ analogues in insulin-dependent diabetes mellitus and other autoimmune diseases: a therapeutic perspective. Diabetes Metab Rev 12: 57-68

9. Rigby WF, Yirinec B, Oldershaw RL, Fanger MW (1987) Comparison of the effects of 1,25-dihydroxyvitamin $\mathrm{D}_{3}$ on T lymphocyte subpopulations. Eur J Immunol 17: 563-566

10. Rigby WF (1988) The immunobiology of vitamin D. Immunol Today 9: 54-58

11. Rabinovitch A, Skyler JS (1998) Prevention of type 1 diabetes. Med Clin North Am 82: 739-755

12. Mathieu C, Laureys J, Sobis H et al. (1992) 1,25-Dihydroxyvitamin $\mathrm{D}_{3}$ prevents insulitis in NOD mice. Diabetes 41: 1491-1495

13. Mathieu C, Waer M, Laureys J, Rutgeerts O, Bouillon R (1994) Prevention of autoimmune diabetes in NOD mice by 1,25 dihydroxyvitamin $\mathrm{D}_{3}$. Diabetologia 37 : $552-558$

14. EURODIAB Substudy 2 Study Group (1999) Vitamin D supplement in early childhood and risk for Type I (insulindependent) diabetes mellitus. Diabetologia 42: 51-54

15. Fraser DR (1995) Vitamin D. Lancet 345: 104-107
16. Markestad T (1983) Effect of season and vitamin D supplementation on plasma concentrations of 25-hydroxyvitamin $\mathrm{D}$ in Norwegian infants. Acta Paediatr Scand 72: 817-821

17. National Nutrition Council (1994) National Nutrition Council's recommendations for infant feeding (in Norwegian). National Nutrition Council, Oslo

18. Nettleton JA (1993) Are n-3 fatty acids essential nutrients for fetal and infant development? J Am Diet Assoc 93: 58-64

19. Endres S, Ghorbani R, Kelley VE et al. (1989) The effect of dietary supplementation with n-3 polyunsaturated fatty acids on the synthesis of interleukin- 1 and tumor necrosis factor by mononuclear cells. N Engl J Med 320: 265-271

20. Hughes DA, Pinder AC (2000) n-3 polyunsaturated fatty acids inhibit the antigen-presenting function of human monocytes. Am J Clin Nutr 171 [Suppl]: 357S-360S

21. Delvin EE, Salle BL, Glorieux FH, Adeleine P, David LS (1986) Vitamin D supplementation during pregnancy: effect on neonatal calcium homeostasis. J Pediatr 109: 328-334

22. Zeghoud F, Vervel C, Guillozo H et al. (1997) Subclinical vitamin $\mathrm{D}$ deficiency in neonates: definition and response to vitamin D supplements. Am J Clin Nutr 65: 771-778

23. Joner G, Søvik O (1989) Increasing incidence of diabetes mellitus in Norwegian children 0-14 years of age 1973-1982. Diabetologia 32: 79-83

24. EURODIAB ACE Study Group (2000) Variation and trends in incidence of childhood diabetes in Europe. Lancet 355: 873-876

25. Green A, Gale EA, Patterson CC (1992) Incidence of childhood-onset insulin-dependent diabetes mellitus: the EURODIAB ACE study. Lancet 339: 905-908

26. Breslow NE, Day NE (1980) Statistical methods in cancer research, Vol. 1, the analysis of case-control studies. IARC Scientific Publications No. 32. International Agency for Research on Cancer, Lyon

27. Johansson L, Solvoll K, Bjørneboe GE, Drevon CA (1997) Dietary habits among Norwegian men and women. Scand J Nutr 41: 63-70

28. Johansson L, Solvoll K, Bjørneboe GE, Drevon CA (1998) Intake of very-long-chain n-3 fatty acids related to social status and lifestyle. Eur J Clin Nutr 52: 716-721

29. Joner G (1992) The epidemiology of insulin-dependent diabetes mellitus in Norway: incidence, prevalence, microvascular complications and mortality [Thesis]. Haukeland University Hospital, and Aker University Hospital, Oslo

30. Trygg K, Lund-Larsen K, Sandstad B et al. (1995) Do pregnant smokers eat differently from pregnant non-smokers? Paediatr Perinat Epidemiol 9: 307-319 